\title{
Austrian Experience with Lixisenatide Under Real-Life Conditions: A Prospective Observational Study
}

Helmut Brath · Heidemarie Abrahamian - Tvrtko Karuza •

Roman Mihaljevic · Martin Pfohl

Received: October 17, 2018 / Published online: January 17, 2019

(C) The Author(s) 2019

\section{ABSTRACT}

Introduction: Lixisenatide has been studied extensively in randomized clinical trials; however, data on its use in the real-life practice are scarce.

Methods: This study was a prospective, 26-week, multicenter, observational study conducted in Austrian diabetes centers and officebased practices to evaluate efficacy and safety of lixisenatide under real-life conditions in patients with type 2 diabetes.

Results: Out of 144 patients (mean BMI $36.4 \mathrm{~kg} / \mathrm{m}^{2}$, disease duration 12.4 years), 113 completed the documentation at 6 months and $42 \%$ received basal insulin with or without oral antidiabetic drugs. The HbA1c declined from

Enhanced Digital Features To view enhanced digital features for this article go to https://doi.org/10.6084/ m9.figshare.7467278.

\section{H. Brath $(\bowtie)$}

Diabetes Outpatient Clinic, Health Centre South, 1100 Vienna, Austria

e-mail: helmut.brath@wgkk.at

H. Abrahamian

Sozialmedizinisches Zentrum Baumgartner Höhe

Otto-Wagner-Spital, 1140 Vienna, Austria

T. Karuza · R. Mihaljevic

sanofi-aventis GmbH Austria, 1220 Vienna, Austria

M. Pfohl

Ev. Krankenhaus Bethesda, 47053 Duisburg,

Germany
$8.7 \%(72 \mathrm{mmol} / \mathrm{mol})$ to $7.9 \%(63 \mathrm{mmol} / \mathrm{mol})$ and at study end $24.8 \%$ of the patients reached an HbA1c level below 7\%. Fasting and postprandial glucose after lixisenatide administration were reduced by $27 \pm 58 \mathrm{mg} / \mathrm{dl}$ and $45 \pm 67 \mathrm{mg} / \mathrm{dl}$, respectively. At study end body weight $\quad(-4.5 \pm 5.4 \mathrm{~kg}), \quad$ triglycerides $(-10.8 \pm 105 \mathrm{mg} / \mathrm{dl})$, systolic blood pressure $(-4.8 \pm 17.1 \mathrm{mmHg})$, and LDL cholesterol $(-3.7 \pm 25 \mathrm{mg} / \mathrm{dl})$ were reduced. The most commonly reported adverse events were gastrointestinal disorders (18.8\%). Forty-three patients (30\%) discontinued prematurely, mostly caused by lack of efficacy, occurrence of gastrointestinal disorders, and missing reimbursement. The average dose of insulin decreased slightly by 1.5 units (from 29.4 to 27.9).

Conclusion: Lixisenatide demonstrated a similar efficacy and safety profile under real-life conditions as previously shown in randomized clinical trials.

Funding: sanofi-aventis GmbH Austria.

Keywords: HbA1c; Lixisenatide; PATIO; Reallife data; Short-acting GLP-1 receptor agonists; Type 2 diabetes

\section{INTRODUCTION}

The introduction of GLP-1 receptor agonists (GLP-1 RA) has brought an attractive treatment 
addition to the therapeutic armamentarium for type 2 diabetes (T2DM), being the first generation of antidiabetic drugs with a significant positive effect on weight and without evidence for increased risk of hypoglycemia. Although the incretin effect was first reported in the 1980s, it took more than two decades until the first GLP-1 RA was developed for therapeutic use in $2005[1,2]$. Nowadays, the significance of this drug class has become indisputable and is reflected in the current position statement of the American Diabetes Association/European Association for the Study of Diabetes, where its use is recommended in nearly all stages of the disease [3]. Owing to the beneficial findings in some of the recent cardiovascular outcome trials [4], it can be assumed that their use in clinical practice will increase in the future.

While all members of the GLP-1 RA class are united by their activity in tissues carrying GLP-1 receptors-including pancreas, gastrointestinal tract, muscle, heart, brain, kidney, and adipose tissue-a sub-classification of GLP-1 RA based on their pharmacological and clinical properties into short- and long-acting groups seems generally recognized $[5,6]$. The long-acting members like liraglutide, exenatide QW, dulaglutide, albiglutide, or semaglutide continuously act on the GLP-1 receptor which leads to more sustainable reductions of fasting plasma glucose (FPG) and HbA1c when compared to the shortacting class representatives [5, 7]. At present, exenatide BID and lixisenatide are the only two available drugs within the short-acting group. Their mechanism of action is primarily deceleration of the gastric emptying, which leads to the reduction of postprandial glucose (PPG) excursions $[6,8]$ and thereby reduces post meal insulin expositions $[9,10]$.

Lixisenatide became commercially available in Austria in 2014, but ever since its use has been limited exclusively to centers of excellence, including restrictions to a certain subset of patients (e.g., with a body mass index (BMI) greater than 30 and preceding use of DPP-4 inhibitors). The drug has been extensively studied during the pivotal clinical GetGoal program, where its efficacy and safety in combination with oral antidiabetic drugs (OADs) and with basal insulin (BI) have been established [11]. In addition, the ELIXA outcome trial has confirmed the cardiovascular safety of the drug in a population of patients with recent acute coronary syndrome [12].

In contrast to randomized clinical trials, realworld studies play a crucial role in evaluating treatment effectiveness, especially in large heterogeneous patient populations, without stringent inclusion and exclusion criteria or other aspects that limit generalizability $[13,14]$. However, only a few reports from real-world studies with lixisenatide have emerged recently [15-17]. Therefore we conducted an observational study called PATIO (Prandial Adding Therapy to Insulin or OAD) in order to better understand the efficacy and safety of the drug under real-life conditions, specifically under the terms of the reimbursement rules of this drug in Austria and to evaluate whether the study results reflect those previously observed in large randomized clinical trials.

\section{METHODS}

\section{Study Design}

PATIO was a prospective, open-label, observational, multicenter study in Austria conducted in patients with type 2 diabetes treated in an outpatients setting. As required by the Austrian Medicines Act, this type of observational study allows only for documentation of data according to clinical practice and within the drug label. Moreover participating centers neither received specific instructions from any party nor conducted additional diagnostic or therapeutic interventions, besides those that were performed routinely anyway. The study protocol was approved by local ethic committees as applicable and the Austrian Ministry of Health was notified of study conduct (registration number NIS004164; https://forms.ages.at/nis/ listNis.do). This study was reviewed and approved under protocol EK 14-247-VK-NIS by the Ethic Committee of the City of Vienna, protocol EK Nr. 1072/2015 by the Medical University of Vienna, EK-Votum 02/2014 by the Austrian Working Group for Clinical Pharmacology and Therapy and was performed in 
accordance with the ethical standards of the 1964 Declaration of Helsinki. Informed consent was obtained from all individual participants included in the study.

\section{Data Collection and Management}

The selection of centers aimed to ensure equal geographic distribution as well as representation of office-based and clinic specialists as well as academic and non-academic institutions. Participating physicians received case report forms (CRF) and training in reporting of adverse events (AEs) by the study sponsor. Data collection was performed during routine visits in a paper-based form. The patients were identified on the documentation sheets by means of code (pseudonymized data collection). The type of the collected patient data included demographics, history of the disease, glycemic and lipid parameters, timing of injection, concomitant antidiabetic medication, and safety data with special focus on hypoglycemic events and gastrointestinal disorders.

\section{Patient Population and Study Medication}

The aim of the study was to evaluate the efficacy, safety, and tolerability of lixisenatide with respect to the use of the drug either in combination with OADs alone or with basal insulin. Patients were eligible for study after they have signed informed consent and fulfilled the following criteria: type 2 diabetes, age of 18 or older, $\mathrm{HbA} 1 \mathrm{c}$ greater than $7.0 \%$, history of use of DPP-4 inhibitors in the highest tolerated dose for at least 3 months and/or use of basal insulin for at least 3 months (according to the Austrian reimbursement rules, all patients had to have one of the five DPP-4 inhibitors registered in Austria before intensifying to a GLP-1 RA, i.e., sitagliptin, vildagliptin, saxagliptin, alogliptin, or linagliptin), BMI greater than $30 \mathrm{~kg} / \mathrm{m}^{2}$, GLP1 RA naive, and able to conduct self-measured plasma glucose (SMPG) monitoring. On the other hand, patients were ineligible if their treatment regimen continued or planned to include DPP-4 inhibitors, sulfonylureas, glinides, if they had or there was a plan to establish a mealtime insulin or if they had reduced renal function (estimated glomerular filtration rate below $45 \mathrm{ml} / \mathrm{min}$ ), history of acute or chronic pancreatitis, severe gastrointestinal disease, diagnosis of cancer within the last 5 years (or pancreas carcinoma at any time), or any other life-threatening disease. Patients were asked to provide documentation on glucose levels either in a 4- or 7-point SMBG profile. Measurements were carried out by patients' own glucometer devices. Confirmed and confirmed nocturnal symptomatic hypoglycemic events were defined as blood glucose concentrations of $70 \mathrm{mg} / \mathrm{dl}$ or less. Severe hypoglycemia was defined as an episode in which a patient required help from another person and addition of glucose was required or a measured blood glucose concentration of $54 \mathrm{mg} / \mathrm{dl}$ or less was indicated.

Lixisenatide (Lyxumia ${ }^{\circledR}, \quad$ sanofi-aventis groupe, Paris) was given subcutaneously once daily $60 \mathrm{~min}$ before the main meal with the starting dose of $10 \mu \mathrm{g}$ in the first 14 days and then increased to $20 \mu \mathrm{g}$ after that. The therapy was documented for up to approximately 6 months. The three study visits were scheduled for baseline, then approximately 2 (week 6-10) and 6 months (week 22-30) after the study began.

\section{STUDY OUTCOMES}

The primary outcome parameter of the study was change of $\mathrm{HbA1c}$ in the total population as well as in both subpopulations of interest (basal insulin and OADs) from baseline to visit 3 . The secondary outcome parameters included change in fasting and postprandial glucose after lixisenatide administration, the response rate (patients with HbA1c below 7.0\%, or achieving their individual target value defined by the investigator, or achieving a combined endpoint defined by HbA1c below 7.0\%, no occurrence of hypoglycemic events, and no weight gain), change of weight, waist circumference, and BMI at all visits, use and type of OADs and BI at baseline and at the end of the study, occurrence of hypoglycemic events and gastrointestinal adverse effects, including early withdrawals due to adverse effects. 
Table 1 Baseline characteristics of the patients

\begin{tabular}{llll}
\hline Mean $(\mathbf{S D})$ & OAD group $(\boldsymbol{n}=\mathbf{8 4})$ & Basal insulin group $(\boldsymbol{n}=\mathbf{6 0})$ & Total $(\boldsymbol{n}=\mathbf{1 4 4})$ \\
\hline Age, years & $58.3(9)$ & $64.0(10)$ & $60.7(9.8)$ \\
Female sex, $n(\%)$ & $34(40)$ & $40(67)$ & $51.4(74)$ \\
Weight, kg & $109.7(20.6)$ & $100.9(17.2)$ & $106(19.7)$ \\
BMI, kg/m ${ }^{2}$ & $37.0(5.6)$ & $35.5(5.1)$ & $36.4(5.4)$ \\
Waist circumference & $121.3(14.6)$ & $119.2(13.4)$ & $120.4(14.1)$ \\
Disease duration, years & $10.3(5.9)$ & $15.4(9.9)$ & $12.4(8.1)$ \\
Duration of insulin therapy & & $4.9(6.1)$ & \\
HbAlc & $8.9(2.4)$ & $8.5(1.4)$ & $8.7(2.0)$ \\
FPG (mg/dl) & $190.4(53.0)$ & $169.4(55.1)$ & $181.8(54.7)$ \\
PPG (mg/dl) & $215.4(59.2)$ & $230(68.4)$ & $221.9(63.4)$ \\
Triglycerides & $208.4(105.5)$ & $178.3(101.2)$ & $196.1(104.5)$ \\
HDL cholesterol & $45.4(11.6)$ & $47.7(12.6)$ & $46.3(12)$ \\
LDL cholesterol & $96.4(42.9)$ & $91.5(33.8)$ & $94.4(38.5)$ \\
Total cholesterol & $176.5(42.6)$ & $171.9(42)$ & $174.6(42.3)$ \\
\hline
\end{tabular}

\section{Statistical Considerations}

PATIO was an observational, open-label study without comparator arm. The study was descriptive in nature and did not involve the testing of any pre-formulated hypotheses. Thus, no formal calculation of statistical power was carried out. Nonetheless the following statistical precision could have been achieved for the mean reduction in the HbA1c value (measured in percent): for a sample size of 300 patients, assuming a standard deviation of $1.1 \%$, the length of the two-sided 95\% confidence interval for the mean decrease was $0.25 \%$; in a subgroup of 150 patients the length of the $95 \%$ confidence interval was $0.35 \%$. For an assumed hypoglycemia rate of $25 \%$, the length of a two-sided 95\% confidence interval was $10.1 \%$ for 300 and $14.2 \%$ for 150 patients, respectively. A lower hypoglycemia rate of $15.0 \%$ would translate into $8.2 \%$ for 300 and $11.8 \%$ for 150 patients.

\section{RESULTS}

Between September 2014 and December 2016 a total of 144 patients were enrolled in the study in 23 centers. Of those, 138 patients were documented during visit 2 , carried out on average 57 days after visit 1 , and 113 were followed up until visit 3 , which was carried out on average 174 days after visit 1 . The most frequently used oral drug at the study entry was metformin (73.6\%) and $41.7 \%$ of the patients received basal insulin. Of those, the majority of 37 patients (61.7\%) used human NPH insulin and 23 (38.3\%) used long-acting insulin analogues. Basal insulin treatment was ongoing and documented in 48 patients $(42.5 \%)$ at visit 3 . The total insulin dose showed a moderate decrease from 29.4 to 27.9 units throughout the study.

A BMI greater than $30 \mathrm{~kg} / \mathrm{m}^{2}$ was noticed in $139(97.2 \%)$ patients. The mean weight was $117 \pm 18 \mathrm{~kg}$ in men and $101 \pm 16 \mathrm{~kg}$ in women. At baseline, the mean disease duration was $11 \pm 7$ years in men and $14 \pm 9$ years in 
women. The individually defined HbA1c target values were $7.1 \% \pm 0.5$. Baseline characteristics of the patients are presented in Table 1.

After lixisenatide was prescribed independently of the study, patients could be enrolled. A total of 93 patients $(65 \%)$ continued lixisenatide until the end of the study. In 43 patients (30\%), discontinuation was reported on average after $125 \pm 68$ days. The main reasons for discontinuation included lacking effectiveness (19 cases, $13.2 \%$ ) and adverse events (9 cases, 6.3\%). In patients who received insulin, the drug had been applied for $4.9 \pm 6.1$ years. At all visits, lixisenatide was injected before breakfast in $61 \%$, lunch in $19 \%$, and dinner $21 \%$ of the cases.

Furthermore, all patients received concomitant medication during the study. Most medications were classified as ATC-C (cardiovascular medications, 101 prescriptions) and ATC-A (alimentary tract and metabolism, 89 prescriptions). However, besides diabetes therapies, concomitant medication was collected only in case of AEs, thus allowing for incomplete recording.

\section{Effectiveness}

Among patients who completed the study the following changes were observed from baseline to month 6 (Fig. 1).

Average HbA1c in the 112 patients who completed all three study visits declined by $0.7 \pm 1.6 \% ; p<0.0001$ (for total population from $8.7 \% \quad(72 \mathrm{mmol} / \mathrm{mol})$ to $7.9 \%$ (63 mmol/mol), Fig. 2). The proportion of patients participating in visit 3 who achieved HbA1c below $7.0 \%$ was $24.8 \%(n=28)$. Patients with BI showed slightly higher response rates $(27.1 \%)$ than OAD patients $(23.1 \%) ; p=0.664$ between groups. The combined endpoint of HbA1c below $7.0 \%(53 \mathrm{mmol} / \mathrm{mol})$, with no weight gain, and no hypoglycemia was achieved by $18.1 \%$ of all patients and $22.9 \%$ of the patients participating in visit 3. More patients with a disease duration of up to 10 years achieved $\mathrm{HbA} 1 \mathrm{c}$ below $7.0 \%$ and the combined endpoint when compared to patients

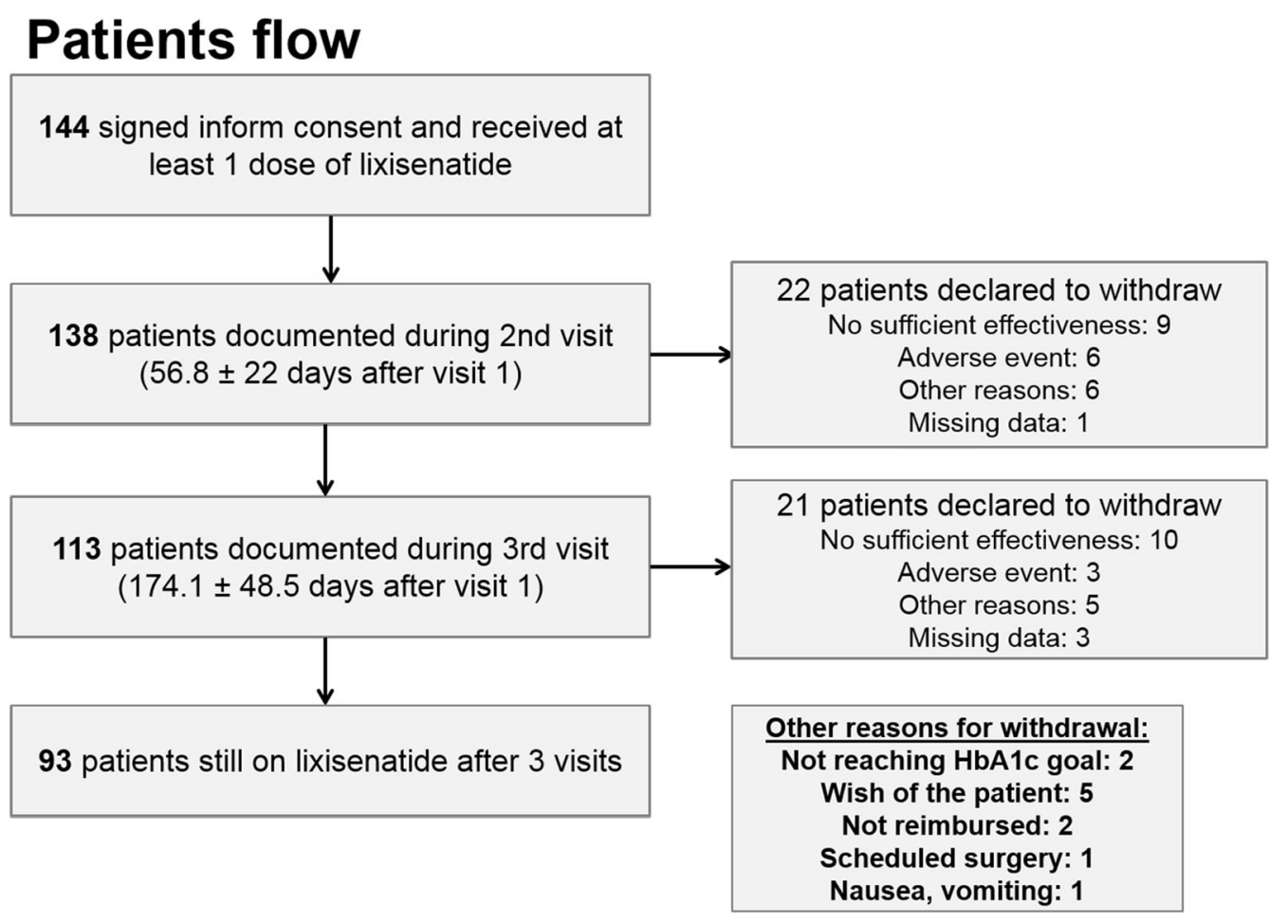

Fig. 1 Patients flow diagram in the study 

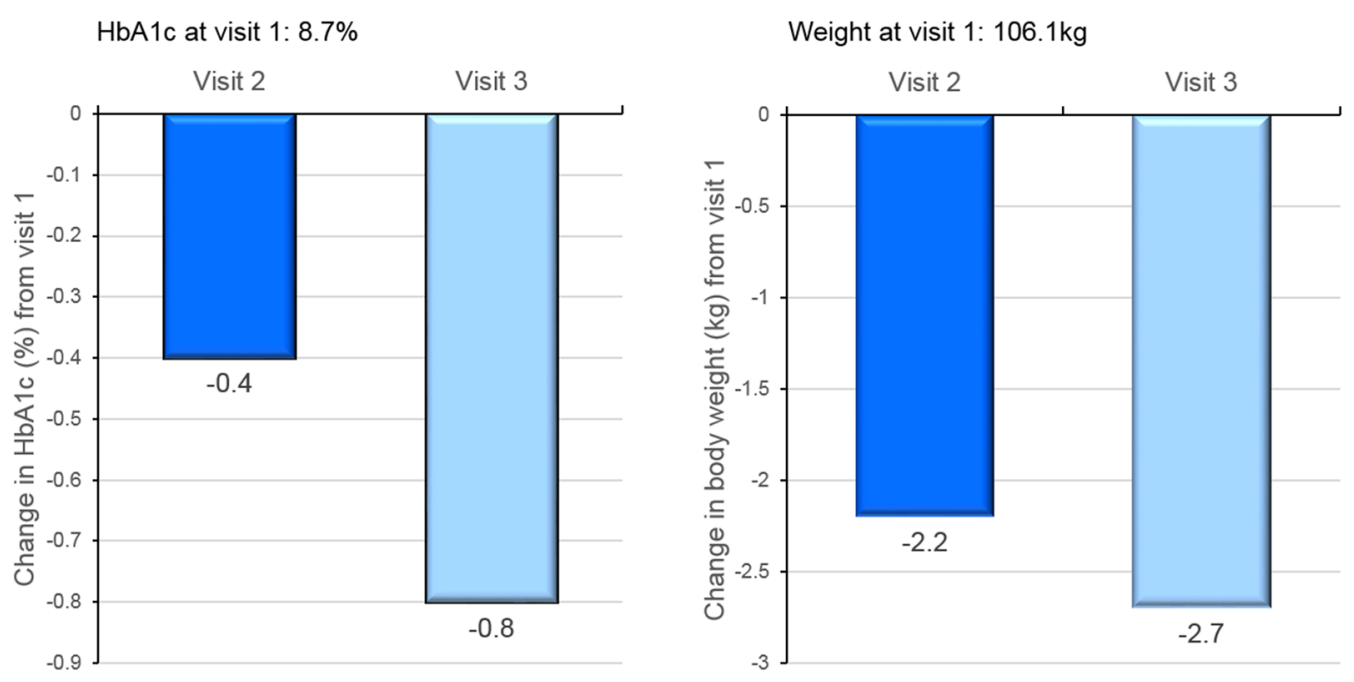

Fig. 2 Change in HbAlc and weight during the study
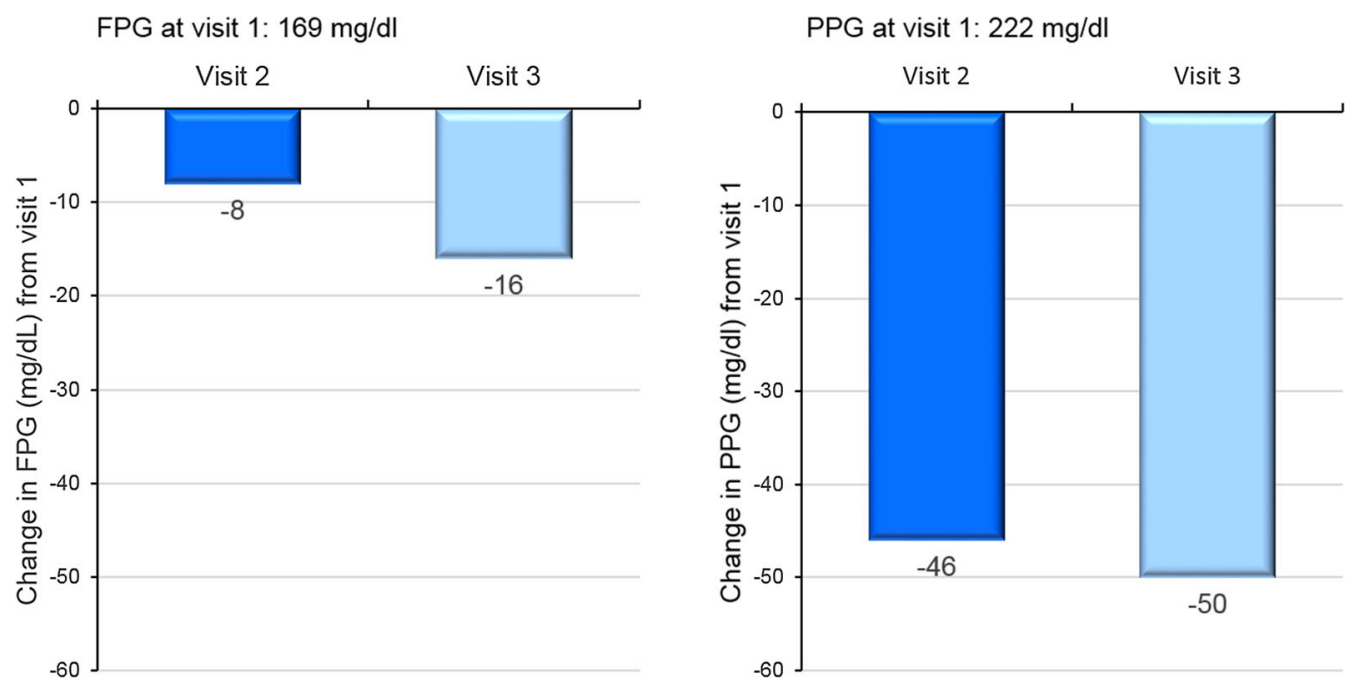

Fig. 3 Change in fasting plasma glucose (FPG) and postprandial plasma glucose (PPG) during the study

with a disease history of more than 10 years ( $32.7 \%$ vs. $19.2 \%$ and $29.1 \%$ vs. $19.2 \%$ ).

Fasting and postprandial blood glucose dropped on average by $27 \pm 58 \mathrm{mg} / \mathrm{dl}$ $(p<0.0001)$ and $45 \pm 67 \mathrm{mg} / \mathrm{dl}(p<0.0001)$, respectively (for changes in total population, see Fig. 3; for 7-point SMBG profiles, see Fig. 4). The median daily swings (peak-trough) of glucose concentrations were also diminished by $26 \pm 46(p<0.0001 ;$ from $103 \pm 51 \mathrm{mg} / \mathrm{dl}$ at visit 1 to $78 \pm 42 \mathrm{mg} / \mathrm{dl}$ at visit 3$)$.
Body weight decreased throughout the study by $4.5 \pm 5.4 \mathrm{~kg}(p<0.0001)$. In the BI group, the decrease was $4.0 \pm 5.3 \mathrm{~kg}$ and in the OAD group the decrease was $4.9 \pm 5.5 \mathrm{~kg} ; p<0.0001$ respectively and $p=0.386$ between groups, without gender-specific differences (for changes in total population, see Fig. 2). The number of obese patients (BMI $30 \mathrm{~kg} / \mathrm{m}^{2}$ or higher) dropped from $97 \%$ (total population) to $83 \%$ in the BI group and from $98 \%$ to $88 \%$ in the OAD group. Waist circumference was reduced by 


\section{7-point SMBG profile}

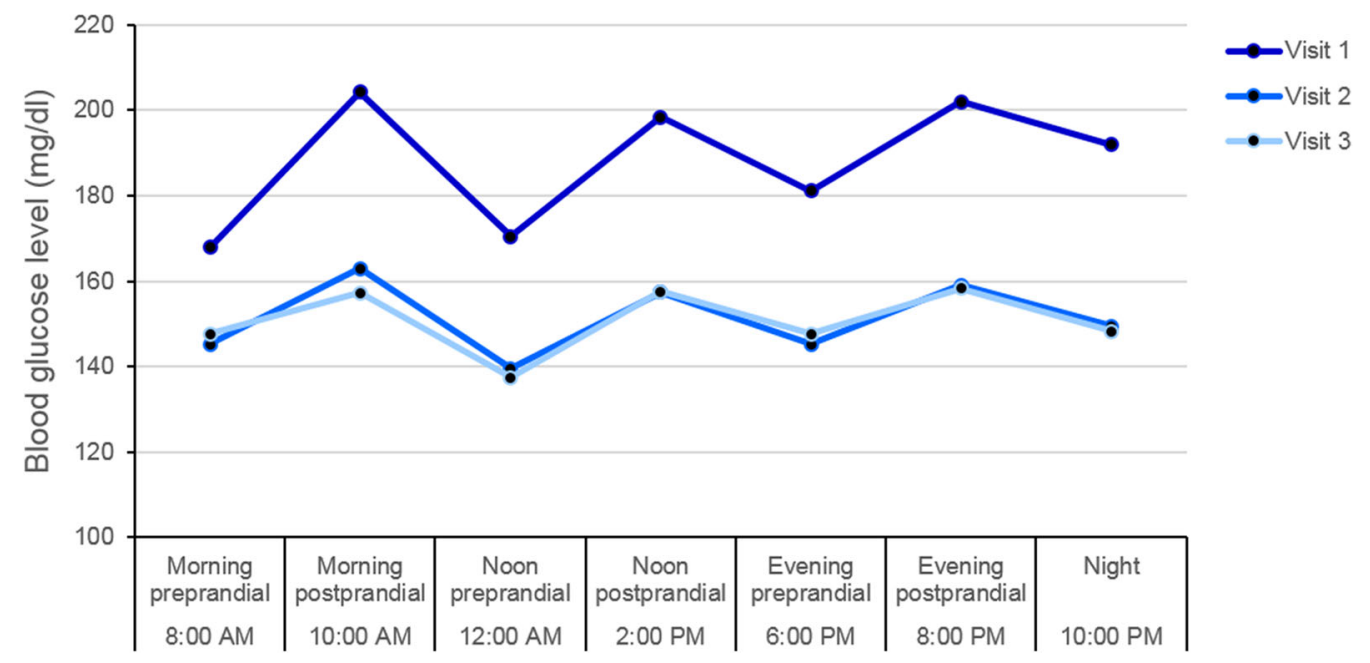

Fig. 4 Mean 7-point SMBG profiles from visit 3 to visit 5

$3.6 \pm 4.7 \mathrm{~cm}(p<0.0001)$, with small differences between genders or treatment groups.

Systolic blood pressure decreased by $4.8 \pm 17.1 \mathrm{mmHg}$ ( $p=0.0066$; for total population from $140.5 \pm 18$ to $135 \pm 16 \mathrm{mmHg}$ ). A numerically smaller reduction of $2.3 \pm$ $10.6 \mathrm{mmHg}(p=0.0342)$ was reported for diastolic blood pressure (for total population from $83 \pm 11$ to $81 \pm 9 \mathrm{mmHg}$ ).

With respect to laboratory parameters, patients showed a decrease in triglycerides by $10.8 \pm 105.4 \mathrm{mg} / \mathrm{dl}(p=0.3504)$. The reduction appeared stronger in women $(-18.7 \pm$ $126.4 \mathrm{mg} / \mathrm{dl} ; \quad p=0.3552)$ than in men $(-3.5 \pm 82.6 \mathrm{mg} / \mathrm{dl} ; \quad p=0.78) ; \quad p=0.51$ between gender groups. HDL cholesterol remained nearly constant with a slight increase of approximately $1.2 \pm 9.2 \mathrm{mg} / \mathrm{dl}(p=0.2353)$. LDL cholesterol, on the other hand, was reduced by $3.7 \pm 25.2 \mathrm{mg} / \mathrm{dl} \quad(p=0.2045)$. Interestingly, in women a decrease of $9.7 \pm 28.7 \mathrm{mg} / \mathrm{dl} \quad(p=0.0536) \quad$ was observed compared to men, where even a slight increase of $1.4 \pm 20.9 \mathrm{mg} / \mathrm{dl} \quad(p=0.67)$ was found $(p=0.0555$ between gender groups). This was also true for total cholesterol, where the decrease was predominantly observed in the female patients $(-5.7 \pm 44.7 \mathrm{mg} / \mathrm{dl} ; \quad p=$ 0.4249 ), and virtually no change was found in male patients $(0.1 \pm 24.6 \mathrm{mg} / \mathrm{l} ; \quad p=0.98)$; $p=0.458$ between gender groups.

\section{Safety Parameters}

AEs were generally rare with a total of 77 events that affected 48 patients. Most frequently, patients experienced gastrointestinal disorders (27 patients, $18.8 \%$ ), followed by general disorders and administration site conditions (9 patients, 6.3\%). All other classes of adverse events affected less than $5 \%$ of the patients. Two events resulted in hospitalization and were classified as serious, one due to an accident resulting in rib fracture, and one involving syncope and vomiting. Only the latter was assessed as related to the study medication. Overall, a causal relationship to the medication was excluded for 13 AEs (16.9\%). The majority of AEs (50 events, 64\%) were classified as "mild", 22 events $(28.6 \%)$ were assessed as "moderate", and $5(6.5 \%)$ as "severe". The actions taken with regard to the study medication involved no change (37 AEs, 48.1\%) and withdrawal (33 cases, $42.9 \%)$.

Gastrointestinal discomfort was reported by 7 patients $(4.9 \%)$ at visit 1, by $23(16.8 \%)$ at visit 2 , and by 5 patients $(4.4 \%)$ at visit 3 (Fig. 5). Throughout the study, men were more affected than women. The most abundant symptom of gastrointestinal discomfort was nausea, followed by vomiting and diarrhea. No case of pancreatitis was reported during the study. 


\section{Gastrointestinal disturbances documented at visit 2 and 3}

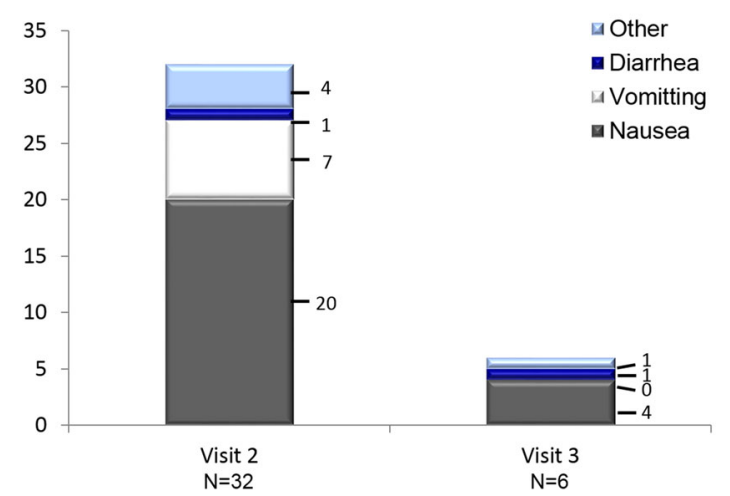

Fig. 5 Gastrointestinal disturbances reported at visit 2 and visit 3

A small proportion of patients were affected by hypoglycemic events, which were recorded in 5 patients $(3.5 \%)$ prior to visit 1 , in 3 patients $(2.2 \%)$ at visit 2 , and not at all at visit 3 . Only one hypoglycemic event between visit 1 and 2 occurred at night, and one event prior to visit 1 was severe. No severe nocturnal hypoglycemia events were reported. Hypoglycemic events appeared to be more frequent in patients treated with OADs; however, the number of events was too low for a meaningful analysis.

\section{DISCUSSION}

The magnitude of effects of a drug in regular clinical practice potentially may differ from those reported in randomized clinical trials [18]. In this context, however, our study has confirmed the already well-defined clinical profile of lixisenatide. At the end of our study, $24.8 \%$ of patients receiving therapy achieved HbA1c levels below $7.0 \%$, which echoes the response rates seen in the intention-to-treat (ITT) analysis of the GetGoal program with lixisenatide, but exceeds the ones recently reported in a reallife study, where lixisenatide was compared with liraglutide [16]. Whereas the higher percentage of patients reaching an HbA1c goal below $7 \%$ can be explained by the lower baseline HbA1c level in our study $[8.7 \%$ $(72 \mathrm{mmol} / \mathrm{mol})$ vs. $9.5 \%(80 \mathrm{mmol} / \mathrm{mol})]$, it is noteworthy that the reduction of $\mathrm{HbA1c}$ of about $0.7 \%$ is comparable in both studies, despite the lower HbA1c in this study. Of note, all patients in our study had a pre-treatment with a DPP-4 inhibitor, which was stopped at the initiation of lixisenatide, underscoring the effectiveness of lixisenatide even after use of oral incretins. Positive influences of lixisenatide on blood lipids and blood pressure were also in line with previous findings. Note that the observed mean weight loss of $4 \mathrm{~kg}$ in our reallife setting was stronger than in the GetGoal studies [11], possibly because of a higher BMI at baseline as well as analyses of patients who completed the treatment.

In terms of patient population, more than half of the patients in PATIO were not on basal insulin yet, although average HbA1c before study entry was $8.9 \%(74 \mathrm{mmol} / \mathrm{mol})$. This finding overlaps with results from a previous multinational study [19] and indicates a significant clinical inertia towards start of insulin therapy in general. Of note, patients already treated with basal insulin were also far away from their anticipated HbA1c goals, despite being on insulin treatment for nearly 5 years. Different reasons, not necessarily related to the type of insulin used, might have contributed to that fact. Firstly, study patients were receiving rather moderate doses of 29 units/day. Given the high BMI in studied patients of above $35 \mathrm{~kg} /$ $\mathrm{m}^{2}$, it is doubtful that the chosen insulin dose was sufficient to control glycemia. Secondly, FPG levels at study entry were around $180 \mathrm{mg}$ / $\mathrm{dl}$ and although moderately reduced during the study $(156.3 \mathrm{mg} / \mathrm{dl}$ and $146.3 \mathrm{mg} / \mathrm{dl}$ for the second and the third study visit), they remained above the recommended target of $130 \mathrm{mg} / \mathrm{dl}$ [20]. The latter finding also suggests a lack of the treat-to-target approach in the cases documented in our study. One can speculate whether the study investigators were aware of the intrinsic moderate impact of lixisenatide on the FPG and the need for basal insulin dose adaptation following lixisenatide initiation. Finally, nearly two thirds of the patients in our cohort used NPH insulin. This high percentage of use of human basal insulin is different from the patient populations in multinational studies conducted with lixisenatide in combination 
with insulin glargine. This difference arises because the Austrian health insurance system only reimburses human insulin for the first-line basal insulin therapy. The costs for long-acting insulin analogues are covered only in the case of recurrent night-time episodes of hypoglycemia by the therapy with NPH insulin. The large use of NPH in addition to the clinical inertia to increase basal insulin doses may explain why patients were not at FPG target during the study.

In light of some earlier research, it could be assumed that the effect on prandial control becomes generally more "visible" with lower HbA1c values [21, 22]. In the present study average postprandial blood glucose after lixisenatide administration was reduced from $222 \mathrm{mg} / \mathrm{dl}$ at visit 1 to $172 \mathrm{mg} / \mathrm{dl}$ at visit 3, meeting the target for postprandial glucose of below $180 \mathrm{mg} / \mathrm{dl}$, defined by the ADA [23]. Given the mechanism of action of lixisenatide, the combination with basal insulin pursues complementary targets and appears to be a very logical treatment approach. The concept of basal and prandial control in the context of lixisenatide has already been proven therapeutically successful in several randomized clinical trials of either combination of free components [24-26] or fixed-ratio combination [27, 28]. However, those were large interventional studies and followed the typical treat-to-target formula, which was not applied in our study.

The relatively high discontinuation rate in our study of $30 \%$ (43 patients) had multiple causes; however, the one most commonly reported reason was "lack of efficacy" in a total of 21 cases. In this context it needs to be mentioned that the study protocol has not clearly predefined that term, but left it to the discretion of the investigator as a result of the observational nature of our study. Also, as defined by the legal framework in Austria, the supply of study medication by the sponsor was not permitted for observational studies. Thereby physicians had to provide details of the clinical status for every single treatment course after a few weeks of initial treatment with lixisenatide in written form and apply for reimbursement to the health insurance provider and this administrative workload may have limited the lixisenatide treatment refill. Only seven patients reported a discontinuation due to adverse events. Other reasons included the patients' wishes [15], no reimbursement of the drug [11], and scheduled surgery [3].

Our study has several limitations including its open-label character, lack of comparator arm, relatively small total number of patients included who completed the evaluation, and finally the quite heterogeneous population as a part of the effort to reflect clinical reality. The participants in the study tend to have higher BMIs than the general population and selected centers were perhaps more experienced in conducting studies than the rest of the physicians in the country, thereby shrinking the generalizability of the results to the entire population. Furthermore, subgroups in our study were too small to clearly identify trends or differences in response, so larger studies will be needed to follow any hypotheses potentially originating from PATIO. The very few reported episodes of hypoglycemia, which probably reflected the generally high HbA1c levels of patients enrolled, the relatively low dose of basal insulin, and exclusion of patients with sulfonylureas or glinides. However, the events could also be underreported, a common issue in real-life studies.

\section{CONCLUSION}

Lixisenatide showed in our study a similar efficacy and safety profile under real-life conditions as already demonstrated in randomized clinical trials. Educational efforts towards identifying appropriate patients with the need for postprandial glucose control have potential to further improve outcomes with lixisenatide in routine clinical practice.

\section{ACKNOWLEDGEMENTS}

The authors thank the participants of the study, the clinical research organization, Dr. Robert Heinz \& Partner GmbH for Data Management and Clinical Study Report. 
Funding. Sponsorship for this study and article processing charges were funded by sanofi-aventis GmbH Austria. All authors had full access to all of the data in this study and take complete responsibility for the integrity of the data and accuracy of the data analysis.

Authorship. All named authors meet the International Committee of Medical Journal Editors (ICMJE) criteria for authorship for this manuscript, take responsibility for the integrity of the work as a whole, and have given final approval to the version to be published.

Authorship Contributions. Sergio Modafferi, Markus Ries, Verena Peters, Vittorio Calabrese, Claus Peter Schmitt, Peter Nawroth, and Stefan Kopf contributed to this article. Sergio Modafferi, Markus Ries, and Verena Peters were involved in planning this article. Vittorio Calabrese, Claus Peter Schmitt, Peter Nawroth, and Stefan Kopf were involved in interpretation of literature, and in drafting and critically revising the manuscript.

Disclosures. Helmut Brath has acted as speaker or advisory board member for Amgen, AstraZeneca, Boehringer Ingelheim, Eli Lilly, Medtronic, MSD, Mylan, Novartis, Novo Nordisk, Pfizer, Sanofi, and Servier. Heidemarie Abrahamian has taken part in advisory panels for Sanofi, Novo Nordisk, Böhringer Ingelheim, Servier, Astra Zeneca, Ely Lilly, Novartis, MSD, Apomedica and has acted as a speaker for Sanofi, Böhringer Ingelheim, Astra Zeneca, Ely Lilly, Ferring, Apomedica, Servier, Menarini. Martin Pfohl has acted as speaker and has taken part in advisory board panels for Sanofi, Novo Nordisk, Böhringer Ingelheim, and Ely Lilly. Roman Mihaljevic is an employee of sanofiaventis GmbH Austria. Tvrtko Karuza is an employee of sanofi-aventis GmbH Austria.

Compliance with Ethics Guidelines. This study was reviewed and approved by the under protocol EK 14-247-VK-NIS by the Ethic Committee of the City of Vienna, protocol EK Nr. $1072 / 2015$ by the Medical University of Vienna, EK-Votum 02/2014 by the Austrian Working Group for Clinical Pharmacology and Therapy and was performed in accordance with the ethical standards of the 1964 Declaration of Helsinki. Informed consent was obtained from all individual participants included in the study.

Data Availability. The datasets generated during and/or analyzed during the current study are available in the registry of Austrian Agency for Health and Food Safety (AGES, https://forms.ages.at/nis/registerDetail.do?id= TkITMDAOMTYO).

Open Access. This article is distributed under the terms of the Creative Commons Attribution-NonCommercial 4.0 International License (http://creativecommons.org/licenses/ by-nc/4.0/), which permits any noncommercial use, distribution, and reproduction in any medium, provided you give appropriate credit to the original author(s) and the source, provide a link to the Creative Commons license, and indicate if changes were made.

\section{REFERENCES}

1. Lund A, Knop FK, Vilsboll T. Glucagon-like peptide1 receptor agonists for the treatment of type 2 diabetes: differences and similarities. Eur J Intern Med. 2014;25(5):407-14.

2. Nauck MA, Homberger E, Siegel EG, et al. Incretin effects of increasing glucose loads in man calculated from venous insulin and C-peptide responses. J Clin Endocrinol Metab. 1986;63(2):492-8.

3. Inzucchi SE, Bergenstal RM, Buse JB, et al. Management of hyperglycaemia in type 2 diabetes, 2015: a patient-centred approach. Update to a position statement of the American Diabetes Association and the European Association for the Study of Diabetes. Diabetologia. 2015;58(3):429-42

4. Marso SP, Daniels GH, Brown-Frandsen K, et al. Liraglutide and cardiovascular outcomes in type 2 diabetes. N Engl J Med. 2016;375(4):311-22.

5. Cornell S. Differentiating among incretin therapies: a multiple-target approach to type 2 diabetes. J Clin Pharm Ther. 2012;37(5):510-24. 
6. Meier JJ. GLP-1 receptor agonists for individualized treatment of type 2 diabetes mellitus. Nat Rev Endocrinol. 2012;8(12):728-42.

7. Htike ZZ, Zaccardi F, Papamargaritis D, Webb DR, Khunti K, Davies MJ. Efficacy and safety of glucagon-like peptide- 1 receptor agonists in type 2 diabetes: a systematic review and mixed-treatment comparison analysis. Diabetes Obes Metab. 2017;19(4):524-36.

8. Meier JJ, Rosenstock J, Hincelin-Mery A, et al. Contrasting effects of lixisenatide and liraglutide on postprandial glycemic control, gastric emptying, and safety parameters in patients with type 2 diabetes on optimized insulin glargine with or without metformin: a randomized open-label trial. Diabetes Care. 2015;38(7):1263-73.

9. Kapitza C, Forst T, Coester HV, Poitiers F, Ruus P, Hincelin-Mery A. Pharmacodynamic characteristics of lixisenatide once daily versus liraglutide once daily in patients with type 2 diabetes insufficiently controlled on metformin. Diabetes Obes Metab. 2013;15(7):642-9.

10. Linnebjerg H, Park S, Kothare PA, et al. Effect of exenatide on gastric emptying and relationship to postprandial glycemia in type 2 diabetes. Regul Pept. 2008;151(1-3):123-9.

11. Trujillo JM, Goldman J. Lixisenatide, a once-daily prandial glucagon-like peptide- 1 receptor agonist for the treatment of adults with type 2 diabetes. Pharmacotherapy. 2017;37(8):927-43.

12. Pfeffer MA, Claggett B, Diaz R, et al. Lixisenatide in patients with type 2 diabetes and acute coronary syndrome. N Engl J Med. 2015;373(23):2247-57.

13. Brath H, Paldanius PM, Bader G, Mathieu C. Relationship between duration of type 2 diabetes and effectiveness of DPP-4 inhibitor versus sulfonylurea as add-on therapy: a post hoc analysis. Diabetes Ther. 2017;8(4):829-36.

14. Sherman RE, Anderson SA, Dal Pan GJ, et al. Realworld evidence-what is it and what can it tell us? N Engl J Med. 2016;375(23):2293-7.

15. Divino V, DeKoven M, Khan FA, Boye KS, Sapin H, Norrbacka K. GLP-1 RA treatment patterns among type 2 diabetes patients in five European Countries. Diabetes Ther. 2017;8(1):115-28.

16. Feher M, Vega-Hernandez G, Mocevic E, et al. Effectiveness of liraglutide and lixisenatide in the treatment of type 2 diabetes: real-world evidence from the health improvement network (THIN) database in the United Kingdom. Diabetes Ther. 2017;8(2):417-31.
17. Fleischmann $\mathrm{H}$, Goke $\mathrm{R}$, Bramlage P. Addition of once daily prandial lixisenatide to basal insulin therapy in patients with type- 2 diabetes results in a reduction of HbA1c as an effect of postprandial glucose lowering. Diabetes Metab Syndr. 2017;11(Suppl 1):S91-S97.

18. Ahren B, Mathieu C, Bader G, Schweizer A, Foley JE. Efficacy of vildagliptin versus sulfonylureas as addon therapy to metformin: comparison of results from randomised controlled and observational studies. Diabetologia. 2014;57(7):1304-7.

19. Khunti K, Damci T, Meneghini L, Pan CY, Yale JF, SOLVE Study Group. Study of Once Daily Levemir $\left(\mathrm{SOLVE}^{\mathrm{TM}}\right)$ : insights into the timing of insulin initiation in people with poorly controlled type 2 diabetes in routine clinical practice. Diabetes Obes Metab. 2012;14(7):654-61.

20. Clodi M, Abrahamian H, Drexel H, et al. Antihyperglycemic treatment guidelines for diabetes mellitus type 2. Wien Klin Wochenschr. 2016;128(Suppl 2):S45-S53.

21. Monnier L, Lapinski H, Colette C. Contributions of fasting and postprandial plasma glucose increments to the overall diurnal hyperglycemia of type 2 diabetic patients: variations with increasing levels of HbA(1c). Diabetes Care. 2003;26(3):881-5.

22. Riddle M, Umpierrez G, DiGenio A, Zhou R, Rosenstock J. Contributions of basal and postprandial hyperglycemia over a wide range of A1C levels before and after treatment intensification in type 2 diabetes. Diabetes Care. 2011;34(12):2508-14.

23. American Diabetes Association. Standards of medical care in diabetes-2017 abridged for primary care providers. Clin Diabetes. 2017;35(1):5-26.

24. Riddle MC, Forst T, Aronson R, et al. Adding oncedaily lixisenatide for type 2 diabetes inadequately controlled with newly initiated and continuously titrated basal insulin glargine: a 24-week, randomized, placebo-controlled study (GetGoal-Duo 1). Diabetes Care. 2013;36(9):2497-503.

25. Rosenstock J, Guerci B, Hanefeld M, et al. Prandial options to advance basal insulin glargine therapy: testing lixisenatide plus basal insulin versus insulin glulisine either as basal-plus or basal-bolus in type 2 diabetes: the GetGoal duo-2 trial. Diabetes Care. 2016;39(8):1318-28.

26. Seino Y, Min KW, Niemoeller E, Takami A, EFC10887 GETGOAL-L Asia Study Investigators. Randomized, double-blind, placebo-controlled trial of the once-daily GLP-1 receptor agonist lixisenatide in Asian patients with type 2 diabetes insufficiently controlled on basal insulin with or without 
a sulfonylurea (GetGoal-L-Asia). Diabetes Obes Metab 2012;14(10):910-7.

27. Aroda VR, Rosenstock J, Wysham C, et al. Efficacy and safety of LixiLan, a titratable fixed-ratio combination of insulin glargine plus lixisenatide in type 2 diabetes inadequately controlled on basal insulin and metformin: the LixiLan-L randomized trial. Diabetes Care. 2016;39(11):1972-80.
28. Rosenstock J, Aronson R, Grunberger G, et al. Benefits of LixiLan, a titratable fixed-ratio combination of insulin glargine plus lixisenatide, versus insulin glargine and lixisenatide monocomponents in type 2 diabetes inadequately controlled on oral agents: the LixiLan-O randomized trial. Diabetes Care. 2016;39(11):2026-35. 